\title{
An in vitro scratch tendon tissue injury model: effects of High Frequency Low Magnitude loading
}

\section{Isaiah Adekanmbi, Nasim Zargar \& Philippa Hulley}

To cite this article: Isaiah Adekanmbi, Nasim Zargar \& Philippa Hulley (2016): An in vitro scratch tendon tissue injury model: effects of High Frequency Low Magnitude loading, Connective Tissue Research, DOI: 10.1080/03008207.2016.1198338

To link to this article: http://dx.doi.org/10.1080/03008207.2016.1198338

Accepted author version posted online: 13

Jun 2016.

Published online: 13 Jun 2016.

Submit your article to this journal $\sqsubset$

Q View related articles $\longleftarrow$

View Crossmark data \lceil 


\title{
An in vitro scratch tendon tissue injury model: effects of High Frequency Low Magnitude loading
}

\author{
Isaiah Adekanmbi ${ }^{1,2}$, Nasim Zargar ${ }^{3}$, Philippa Hulley ${ }^{3}$
}

1. Institute of Biomedical Engineering, Department of Engineering Science, University of Oxford, Oxford, UK

2. Now at Biomedical Engineering Division, School of Engineering, University of Glasgow, Glasgow, UK

3. Nuffield Department of Orthopaedics, Rheumatology and Musculoskeletal Sciences, University of Oxford, Oxford, UK

Corresponding Author:

Dr Isaiah Adekanmbi

Biomedical Engineering Division, School of Engineering, James Watt South Building, University of Glasgow, Glasgow, G12 8QQ, UK. Phone: +44-141-330-3735. e-mail: i adekanmbi@yahoo.co.uk

\section{ABSTRACT}

The healing process of ruptured tendons is suboptimal, taking months to achieve tissue with inferior properties to healthy tendon. Mechanical loading has been shown to positively influence tendon healing. However, High frequency low magnitude (HFLM) loads which have shown promise in maintaining healthy tendon properties, have not been studied with in vitro injury models. Here we present and validate an in vitro scratch tendon tissue injury model to investigate effects of HFLM loading on the properties of injured rat tail tendon fascicles (RTTFs). A longitudinal tendon tear was simulated using a needle aseptically to scratch a defined length along individual RTTFs. Tissue viability, biomechanical and biochemical parameters were investigated before and 7days after culture. The effects of static, $\operatorname{HFLM}(20 \mathrm{~Hz})$, low frequency $(1 \mathrm{~Hz})$ cyclic loading or no load were also investigated. Tendon viability was confirmed in damaged RTTFs after 7 days of culture and the effects of a $0.77 \pm 0.06 \mathrm{~cm}$ scratch on the 
mechanical property(tangent modulus) and tissue metabolism in damaged tendons were consistent, showing significant damage severity compared with intact tendons. Damaged tendon fascicles receiving $\operatorname{HFLM}(20 \mathrm{~Hz})$ loads displayed significantly higher mean tangent modulus than unloaded damaged tendons $(212.7 \pm 14.94 \mathrm{v} 92.7 \pm 15.59 \mathrm{MPa})$, and damaged tendons receiving static loading $(117.9 \pm 10.65 \mathrm{MPa}) . \mathrm{HFLM}$ stimulation maintained metabolic activity in 7day cultured damaged tendons at similar levels to fresh tendons immediately following damage. Only damaged tendons receiving HFLM loads showed significantly higher metabolism than unloaded damaged tendons(RFU-7021 \pm 635.9 v $3745.1 \pm 641.7)$. These validation data support the use of the custom in vitro injury model for investigating the potential of HFLM loading interventions in treating damaged tendons.

Keywords Tendon, Injury, in vitro, Mechanical load, High Frequency, Mechanobiology, Repair Article History

Received 15 March 2016, revised 24 May 2016, accepted 26 May 2016

\section{Introduction}

The influence of mechanical forces in regulating the function of biological structures has been recognised for over a century. In tendons, mechanical loads play a key role in the maintenance of the healthy tissue's biochemical and biomechanical properties [1-3]. This has led to considerable interest in the ability of various mechanical loading interventions to encourage tendon repair [4-8]. In vitro mechanical loading experiments on isolated rat tail tendon fascicles (RTTFs) are useful for understanding relationships in tendon mechanobiology [9-11]. The primary advantage of this model is that the tissue is slender enough not to need a vascular 
component in order to survive for several weeks in vitro. This simplifies the model to response of tendon matrix and tenocytes only, as well as being experimentally straightforward.

Various in vivo and in vitro models have been developed to study the process of repair following tendon injury, from injection with collagenase [12], application of repetitive in vitro fatigue load cycles resulting in the accumulation of micro-tears in tendon tissue to mimic overuse exercise injuries [13] and finally, performing a transverse laceration across the midsection of flexor tendon segments, which is believed to mirror partial (incomplete) tendon ruptures of varying severity. However, models which employ cyclic mechanical loading to introduce tissue injury in tendon specimens [13] are likely to result in poorly controlled, non-reproducible and catastrophic damage in isolated tendon portions.

More recently, Kondratko-Mittnacht, J., 2015 became the first to produce lacerations in the transverse direction of individual rat tail tendon fascicles in vitro by using a razor blade to penetrate $50-70 \%$ of the fascicle width to achieve clinically relevant damage in tendon tissue [14]. However, this damage model is not used as part of a culture system, thus there are no indications about its usefulness in studying mid-long term tendon mechanobiological effects. High Frequency Low Magnitude (HFLM) loading regimes which are characterised by mechanical loading frequencies above $10 \mathrm{~Hz}$, have been shown to counteract effects of bone disease [15-17], and there are indications that similar stimuli may be clinically effective in other musculoskeletal tissues, including tendon [7]. Grigg NL et al 2013, demonstrated that eccentric loading exercises used in the effective treatment of Achilles tendinopathies were linked with high frequency loading[18]. During his study, eleven human male subjects with Achilles tendinopathy and nine control subject without tendinopathy performed ankle rehabilitation exercises involving eccentric and concentric loading of the Achilles tendon. Force plates and 
markers attached to the lower appendages of the subjects along with an 11 camera motion analysis system was used to determine ground reaction force and provide kinematics, kinetic analysis of the Achilles. It was shown that high frequency loads of 9 and $10 \mathrm{~Hz}$ could be observed in both symptomatic and control tendons receiving eccentric exercises, while tendons receiving concentric loads only displayed a frequency peak at $1 \mathrm{~Hz}[18]$.

However, in vitro studies involving stimulation of isolated damaged tendon segments have focused on investigating low frequency and static mechanical loads[17, 19, 20] possibly since such loads reflect those present in tendons during ordinary physical activity. For instance, Tanaka et al applied in vitro cyclic loads at $0.033 \mathrm{~Hz}$ (2cycles/day) on segments of partially lacerated chicken flexor tendons. After 21 days of culture, immunohistological analysis revealed that low frequency cyclic stimulation of the damaged tendons significantly enhanced proliferation and migration of fibroblasts when compared with unloaded lacerated controls [17].

Concomitantly, tendon exposure to high frequency mechanical loads of $30 \mathrm{~Hz}$, for 10x 60 seconds a day for 8 weeks using whole body vibration (WBV) training has been shown to increase patellar tendon cross sectional area by $3.8 \%$ with an accompanied increase in modulus $(0.86 \pm 0.24$ to $0.87 \pm 0.24 \mathrm{GPa})$. [21]. Such a trend has also been highlighted in animal studies which show a significant increase in the cross sectional area and stiffness of rat flexor tendons after mechanical stimulation with $30 \mathrm{~Hz}$ WBV for 5weeks [22].

Animal studies have revealed a positive metabolic response as demonstrated by increased collagen expression in the patellar tendon of rats receiving WBV stimulation at $30 \mathrm{~Hz}$ for $20 \mathrm{~min}$ a day, 5 days a week, for 5 week in comparison with rats receiving low frequency $(3 \mathrm{~Hz})$ stimulation [23]. However, to date studies on HFLM loading of damaged tendon fascicles have not been performed under controlled culture conditions. 
The aim of this study was to validate an in vitro injury model and use it to explore the effects of mechanical loading (HFLM, low frequency, and static) on the properties of damaged tendon fascicles. Elastic properties were measured and the tissue metabolic status was assessed as indicators of tendon health. The hypothesis tested was that in vitro tendon modulus and tissue metabolism would be maximised in damaged tendons receiving HFLM cyclic loading compared with other mechanically loaded tendons and unloaded damaged tendon groups.

\section{Materials and Methods}

\subsection{Tendon fascicles}

Tendon fascicles from the tails of 14 male Sprague Dawley rats (aged 3-5 months), were harvested within 2 hours of sacrifice. Fascicles were separated into one of seven groups: fresh intact and fresh damaged (Fresh-D), unloaded intact (Un-intact), unloaded damaged (Un-D), HFLM cyclic loaded damaged (20Hz-D), low frequency cyclic loaded damaged (1Hz-D), static loaded damaged (Static-D) tendons. The number of fascicles used was 23, 15, 26, 30, 18, 16, for each respective group (Table 1). There were three control groups: fresh intact, fresh damaged and unloaded intact. All fascicles serving as fresh intact or fresh damaged groups were analysed immediately after harvesting or damage. Other groups were cultured for 3-7 days, depending on experiment.

For validation of mechanical and biological damage severity only, each RTTF was dissected into paired segments with one segment serving as damaged group while the other segment served as intact (undamaged) tendon group, figure 6 and 7. 
Validation tests were performed to assess the effect of an in vitro scratch damage on tissue viability, damage size (scratch length), tendon matrix organisation, tendon modulus, and tendon metabolism.

\subsection{Damage model for tendon fascicles}

In vitro tendon injury was achieved by manual application of a carefully controlled $1 \mathrm{~cm}$ scratch using a sterile 23 gauge insulin needle (Microlance, Fisher Scientific UK) and marker dots $1 \mathrm{~cm}$ apart on a petri-dish lid while maintaining fascicle hydration with drops of DMEM (Figure 1). Following application of the scratch damage, both ends of the fascicle handled by the operator were removed using a surgical scalpel, and the fascicle was immediately placed in DMEM, ready for culture and stimulation.

\subsection{Culture system for HFLM loading}

Rat tail tendon fascicles (RTTFs) with target lengths of $100 \mathrm{~mm}$ were cultured and suspended in $50 \mathrm{ml}$ centrifuge tubes (DMEM supplemented with $10 \%$ foetal calf serum and $1 \%$ penicillinstreptomycin, renewed every 3 days), at $37{ }^{\circ} \mathrm{C}$ and $5 \% \mathrm{CO} 2$. An in vitro loading system (IVLS) for application of HFLM loads with magnetic actuation was used, Adekanmbi et al 2013 [24]. The system is capable of applying average peak cyclic loads of $0.03-0.09 \mathrm{~N}$ superimposed on the static load $(0.06 \mathrm{~N})$ at applied cyclic frequency of $1-20 \mathrm{~Hz}$ with an accuracy of $0.003 \mathrm{~N}$. Typical breaking force for tendon fascicles with diameters between $300-500 \mu \mathrm{m}$ may range from 1-4N 


\subsection{Mechanical loading conditions}

Groups (section2.1) of cultured RTTFs were loaded using a magnetic weight of $6 \mathrm{~g}$ (load $0.06 \mathrm{~N}$ ) with either static, low frequency $(1 \mathrm{~Hz})$ or HFLM $(20 \mathrm{~Hz})$ cyclic mechanical loads for 1 hour a day. A frequency of $20 \mathrm{~Hz}$ was used for HFLM loading due to operating limitations of the custom built stimulation device. Outside of the $1 \mathrm{hr}$ stimulation times, the magnet was lowered to the tube base so that the RTTFs were slack. The load-time relationship for each group is shown in figure 2. Mechanically loaded RTTFs received 5 days of stimulation followed by 2 days of rest. During unloading (Un) conditions the stir bar was lowered to the test tube base so that the RTTFs were slack.

\subsection{Viability}

Tissue viability was assessed using a commercial Live Dead staining kit (Molecular Probes, Invitrogen UK). Stained RTTFs were examined using an Olympus EX51 microscope (for cell counts, $\mathrm{n}=2$ per group) with a $5 \mathrm{x}$ objective over a central Region of Interest (ROI; $1.7 \mathrm{~mm} \mathrm{x}$ $1.3 \mathrm{~mm})$.

Post image analysis and quantification of tissue viability (cell counts) was performed using Image $\mathrm{J}$ and a fascicle was defined as viable if $50 \%$ or more of the observed cells in the ROI were living.

\subsection{Multi-Photon Microscopy (MPM)}

Tendon fascicle morphology, following rinsing in PBS, was examined using an inverted confocal laser scanning multi-photon microscope (LSM 710; Carl Zeiss), coupled to a tunable and pulsed femtosecond Chameleon laser (Coherent, UK) operating at 850nm. An oil immersion objective 
$(\mathrm{NA}=1.0 ; 10 \times$ magnification) was used for focusing the excitation beam and for collecting the backward SHG signals which were then directed by a dichroic mirror to an external detector through a narrow band pass filter $(384-447 \mathrm{~nm})$. Images were acquired $(2.6 \times 0.85 \mathrm{~mm}, \mathrm{n}=3)$ across the longitudinal section containing a scratch in each tendon fascicle. The high power, (long-wavelength) laser-light used in multi-photon microscopy, penetrates $200 \mu \mathrm{m}$ in thick tendon sections, avoiding artefacts typically associated with thin sections. This allows for high resolution visualization of the collagen microstructure deep within tendon sections, eliminating the need for staining reagent [25].

\subsection{Tendon Metabolism}

Metabolic activity in RTTFs was measured using Alamar blue reagent (Abd Serotec, Kiddlington UK). Immediately after culture or stimulation, tendon segments were rinsed in DMEM and supplemented with Alamar blue using a 1:10 (v/v) mixture of the dye reagent and culture media. Following 3 hours incubation the relative fluorescence (Relative Fluorescence Units, RFU) was measured using Fluostar Optima spectrofluorometer (BMG Labtech 413-0120, excitation $530 \mathrm{~nm}$, emission $590 \mathrm{~nm}, 570 \mathrm{~nm}$ cut off) as an indicator of tissue metabolic activity.

\subsection{Biomechanical testing}

Tensile tests were performed using a bench top tensile test machine equipped with a $22 \mathrm{~N}$ load cell (Bose Electroforce, Eden Prairie, MN). Assuming circularity, the average fascicle diameter, based on 7 measurements using an optical micrometer (Keyence, Milton Keynes, UK; precision $1 \times 10-5 \mathrm{~mm}$ ), was used to calculate cross sectional area after mechanical stimulation and prior to tensile testing. Only fascicles with diameters in the range of 300-500 $\mu \mathrm{m}$ were selected prior to damage and culture. 
RTTFs were mounted with a grip to grip length of $15 \mathrm{~mm}$ using a custom non-slip gripping system [26]. RTTFs were tested to failure at $0.05 \mathrm{~mm} \mathrm{~s}^{-1}$. Tendon specimens were kept moist by spraying with PBS. Force and displacement data were captured at $10 \mathrm{~Hz}$ for each RTTF and cross sectional area (CSA) in damaged and intact regions of a fascicle was assumed constant. No preconditioning tests were performed prior to testing until failure for all damaged tendon fascicles. Stress-strain data was smoothed using a 50 point moving average filter and the tangent modulus was calculated as a function of strain to estimate the maximum modulus for each fascicle.

\subsection{Statistical techniques}

Statistical tests (Student's t-test and one-way ANOVA analysis) were performed using GraphPad Prism v.5 (GraphPad Software, La Jolla, CA). A paired t-test was used for validation experiments and in all other cases, an unpaired statistical assessment was performed. A post hoc test with Tukey: Comparison on all pairs of test groups were used following assessment with ANOVA.

\section{RESULTS}

\subsubsection{Tendon viability}

The in vitro injury model was characterized using a Live/Dead staining viability assay. Tendon fascicles in Un-intact, Un-D, Static-D, $1 \mathrm{~Hz}-\mathrm{D}$, and 20Hz-D, tendon groups were confirmed to be viable after 7 days of culture, showing similar viability to fresh intact and fresh-D tendon groups. The proportion of live cells measured in cultured tendon groups was $73.4 \%, 66.9,69.6,69.9$, $71.3 \%$, respectively and for fresh tendons $73.8 \%$ (intact), and 74.2\% (fresh-D), (Figure 3). 
Fresh-D fascicles showed regular and aligned arrangement of collagen fibrils in intact regions, and interrupted distribution of collagen fibrils in scratch regions (Figure 4).

Scratch lengths were measured using the 'Ortho' function on Zen2009 light edition software. Measurements of SHG images from three Un-D RTTF taken from the validation tests (figure 4) revealed that scratch lengths were $0.77 \pm 0.06 \mathrm{~cm}$ and the tissue was completely penetrated during application of a scratch damage.

Unloaded damaged RTTFs also demonstrated tightly packed collagen fibrils with parallel alignment and regular crimp pattern in intact regions after 3 days within a culture environment. In contrast damaged regions displayed loose disorganised fibres with disruption of parallel fibril alignment and interruption of crimp organisation (figure 4).

\subsubsection{Biomechanical damage severity}

Application of a $1 \mathrm{~cm}$ scratch damage along the longitudinal length of unloaded tendon segments resulted in a two-fold significant decrease in the tangent modulus $(p<0.05)$, when compared with intact unloaded controls of the same fascicle after 7 days of culture $(88.64 \pm 9.83 \mathrm{v}$

$186.55 \pm 22.4 \mathrm{MPa}$ ), (figure 5). The reduction in modulus immediately after a scratch damage was $35.34 \%$.

\subsubsection{Biological damage severity}

Alamar blue assay provides an indication of net metabolic activity and was used to assess the biological severity of damage in RTTFs. Introduction of mechanical damage resulted in a significant drop in the metabolic activity of tendon segments by $23.80 \%$ immediately after scratch, and a drop of $17.88 \%$ after 7 days of in vitro culture, when compared with unloaded 
intact tendons (figure 6 , table 2$)(\mathrm{p}<0.05$, Student's t-test). There was no significant change in the metabolism of damaged tendon fascicles between day 0 and day 7 of culture.

\subsection{Modulus after loading}

When varying mechanical loading conditions, damaged tendons fascicles HFLM-D tendons displayed significantly higher modulus than Un-D tendons $(\mathrm{p}<0.0001)$, and Static-D tendons $(p<0.0005)$, (Figure 7). Tendons fascicles from 1Hz-D group also displayed significantly higher modulus than Un-intact tendons, but to a lesser extent than HFLM-D tendons $(p<0.05)$. Amongst the mechanically loaded tendon groups, Static-D displayed the lowest modulus compared with Un-D tendons, and no significant differences were found between the two groups.

\subsection{Metabolism after loading}

Amongst mechanically loaded damaged tendon groups, only tendons stimulated at $20 \mathrm{~Hz}$ showed significantly higher metabolism than unloaded damaged tendons $(\mathrm{p}<0.05$, ANOVA). (Figure 8). Fresh intact, Un-intact, and Fresh-D tendons were examined and served as respective control groups. Damaged tendon fascicles loaded at $1 \mathrm{~Hz}(1 \mathrm{~Hz}-\mathrm{D})$ displayed higher metabolism than those receiving static loading (Static-D), and Static-D tendons displayed the lowest metabolism amongst mechanically loaded damaged tendon groups, however these observed differences were not statistically different. Un-D tendons receiving a scratch injury displayed the lowest metabolism of all tendon groups, significantly lower than fresh intact tendons. Metabolic activity was marginally higher in Fresh-D tendon groups than in Unloaded-Intact tendons but this difference was not statistically significant. 


\section{Discussion}

In the present study we report the use of a custom in vitro injury model for investigating effects of various mechanical loading conditions on damaged tendon fascicles. It was demonstrated that in vitro HFLM mechanical loads of $20 \mathrm{~Hz}$ are more effective than static or low frequency $(1 \mathrm{~Hz})$ cyclic loading for preventing deterioration of tendon modulus and tissue metabolism in damaged tendon fascicles. Stimulation at $20 \mathrm{~Hz}$ maintained both modulus and metabolic activity in 7 day cultured damaged tendons at similar levels to fresh tendons immediately following damage. This could be due to either prevention of degradation following injury or repair or both. Our methods did not detect significant metabolic damage immediately after injury but required 1 week in culture for damage to reach significant levels, suggesting a primarily cell based response which time lags the actual physical cut.

Static, low frequency (LF) cyclic loaded and HFLM loaded damaged tendon groups displayed higher tangent modulus than unloaded damaged tendons after 7 days in culture. Only LF and HFLM cyclic loaded damaged tendons displayed significant differences with unloaded damaged tendons indicating that static loads offer the least stimuli for healing of damaged tendons. In the present communication, tendon cell viability, regular ECM matrix architecture, and successful application of a consistent scratch length in damaged tendon fascicles was used to validate the in vitro injury model. Cultured RTTFs remained viable 7 days after application of a scratch damage and loaded damaged cultured fascicles displayed lower viability than freshly damaged tendons but higher tissue viability than unloaded damaged groups after 7 days of culture.

In contrast to this, Kondratko-Mittnacht et al 2015, reported that cell viability was lower in mechanically loaded damaged tendon fascicles in comparison to non-loaded control tendons. 
However the mechanism used for mechanical treatment of each RTTFs (cyclic loading) in the study was short lived (less than 1 minute in total), did not include a culture system, and thus did not allow for factors associated with cellular response and tissue remodelling in damaged tendons. Also, incorrect handling of lacerated tendon fascicles under non-aseptic conditions may have led to the drop in viability after the mechanical treatment period of approximately 20 seconds [14].

Mechanical and biological damage severity were characterised and application of a scratch damage was found to significantly reduce tangent modulus (MPa) by $35.34 \%$ immediately after damage and $52.5 \%$ after 7 days of culture. These results are in agreement with in vitro studies on lacerated flexor tendons which show that the structural stiffness is unfavourably affected by introduction of partial laceration in tendon specimens [27]. Similarly, the percentage difference in tendon metabolism (RFU) between damaged and intact tendons was reduced from $23.8 \%$ at day 0 to $17.88 \%$ by day 7 of culture. This suggests that cellular activity peaks early on in this damage model and declines thereafter and this observation maybe due to early responses of functioning cells working rapidly to repair damaged cells in the scratch region.

Previous in vitro studies have described protocols for creating mechanically significant damage in loaded cultured tendons $[13,17,19]$ however, these systems do not allow for application of HFLM loads onto damaged tendon fascicles. Lergerlotz was one of the first authors to successfully develop a custom in vitro injury model for application of mechanical damage to study effects of loading in cultured tendon fascicles [19], though a similar technique was introduced by Asundi et al in 2007 for producing mechanical damage onto cultured whole tendon organs [28]. Legerlotz reported that cyclic fatigue loading at $1 \mathrm{~Hz}$ for $1 \mathrm{hr}$ and $30 \%$ failure strain caused no significant damage in isolated tendon fascicles but did increase production of collagen 
alpha chain 1 and expression of interleukin-6. Extending the duration of cyclic load to $5 \mathrm{hrs}$ at $30 \%$ failure strain however, or $60 \%$ failure strain for $15 \mathrm{mins}$ was enough to cause significant mechanical damage (reduction in failure stress) compared to unloaded controls [19]. Although production of uncontrolled tendon fascicle damage may reflect events during physiological tendon injury, application of a defined scratch length confers the ability to conveniently characterize experimental tissue damage and establish a relationship between tendon fascicle damage of a known severity and damage tissue adaptation to HFLM loading. We consider our current (newly developed) technique an advancement to the above protocol since introduction of mechanical damage by fatigue cyclic loading may likely produce spontaneous, catastrophic and uncontrolled damage in isolated tendon fascicles. More recently, Kondratko-Mittnacht et al 2015 produced transverse laceration damage in RTTFs by using a razor blade to penetrate $50-70 \%$ of an individual fascicle width [14]. We produced a consistent scratch length of $0.77 \pm 0.06 \mathrm{~cm}$ in individual rat tail tendon fascicles, in spite of an initial target scratch length of $1 \mathrm{~cm}$. The disparity in target and achieved scratch length may be a result of gap closure due to cross-linking of previously disconnected collagen fibrils by glycosaminoglycans which surround sub fascicle structures and are known to be effective in bridging adjacent collagen molecules [27, 29]. Despite this effect of gap closure it can be inferred from the low standard deviations obtained for scratch lengths subsequent to culture that the chosen model allows for accurate and reproducible damage creation in individual RTTFs. We have previously developed a novel in vitro loading system to show that HFLM mechanical loading interventions maintain the properties of healthy tendons [24] however in vitro studies of HFLM loading on damaged tendons have not been performed. Cyclic loading frequencies of $0.2 \mathrm{~Hz}$ using a dog lacerated flexor tendon healing model showed that higher frequency 
mechanical loads $(0.2 \mathrm{~Hz})$ over a period of 3 and 5 weeks increased modulus and ultimate properties significantly over lower frequency loading $(0.0167 \mathrm{~Hz})$ [30]. Reider et al 2015 showed that administration of high frequency mechanical loads of up to $30 \mathrm{~Hz}$ via WBV plates produced small increases in the stiffness (from $1935 \pm 521$ to $2048 \pm 487 \mathrm{~N} / \mathrm{mm}$ ) and modulus (from $0.86 \pm$ 0.24 to $0.87 \pm 0.24 \mathrm{GPa}$ ) of the patellar tendon of human subject, when compared to nonstimulated controls [21] and this reflects the results of this study since, adaptation to the presence and the absence of mechanical loading is believed to be greater in damaged tendons than in healthy tendons [31].

As shown in experiments on tendon modulus, a cyclic loading frequency dependency was demonstrated on damage site metabolic activity and only RTTFs stimulated at $20 \mathrm{~Hz}$ showed significantly higher metabolism than unloaded damaged tendons. Tanaka et al 1995 investigated the effects of low frequency $(0.03 \mathrm{~Hz})$ cyclic tension on the in vitro healing of chicken flexor tendons. They found that cyclic tension enhanced collagen synthesis, and proliferation and migration of fibroblasts [17], and these results are in agreement with our findings on metabolism. Moreover, a positive effect of high frequency loading on metabolic response of rat patellar tendons as demonstrated by increased collagen expression in the patellar has been shown using WBV stimulation at $30 \mathrm{~Hz}$ for 20 min a day, 5 days a week, for 5 week [23] .

The mechanisms by which high frequency low magnitude (HFLM) mechanical loading prevent deterioration of tendon modulus and tissue metabolism in damaged RTTFs more effectively than static and low frequency cyclic loading regimes are beyond the scope of this study however, it is possible that a higher number of low magnitude load cycles per unit interval of time with HFLM loading may provide an increased stimulus in damaged RTTFs when compared with tendons loaded at low frequency. Furthermore, While tenocytes are well documented to respond to 
appropriate mechanical loading with increased anabolic synthesis of matrix and decreased matrix catabolism, it is also possible that mechanical loading increases circulation and perfusion of the culture medium, thus contributing to fascicle health. Testing was carried out over 7 days which is less time than tendons typically take to fully heal. However, the developed system and biological tissue used did not include vascular components critical to long term tendon repair and as such should be appropriately used to improve the test model. Other limitations of this study include lack of data on failure properties of the fascicles, since a number of fascicles mechanically tested did not rupture. Furthermore, noticeable variation was observed in the peak load applied during HFLM loading conditions, from approximately $0.07-0.14 \mathrm{~N}$. This was a limitation of the custom stimulation device.

Future work will aim to assess changes in scratch length with time throughout the speculated gap closure period and if scratch length is affected by loading conditions.

There is evident need for appropriate injury models for tendon research $[32,33]$ since they enable hypothesis driven testing associated with tendon disease and treatment. Here we have shown that the developed custom in vitro injury model is useful for studying mechanobiological effects in damaged tendon fascicles receiving low frequency and HFLM mechanical loading regimes. The findings reported in this study support the use of a custom in vitro injury model in recognising the potential of HFLM loading interventions for treating damaged tendons.

\section{Funding}

This work was funded by a grant from Engineering and Physical Research Council (EPSRC) studentship (IA). 


\section{Acknowledgements}

Professors Mathis Riehle, Matt Dalby, and Elizabeth K Tanner at University of Glasgow are thanked for their assistance in providing laboratory space and equipment for the execution of some of various experiments performed. These supporters had no involvement in carrying out this study, nor in the writing and submitting of this article.

\section{Conflict of interest}

The authors declare that there is no conflict of interest.

\section{REFERENCES}

1. Wang, J., Mechanobiology of tendon. Journal of Biomechanics, 2006. (9)(39): p. 15631582.

2. Yamamoto, E., Tokura, S., Hayashi, K.,, Effects of cyclic stress on the mechanical properties of cultured collagen fascicles from the rabbit patellar tendon. . J Biomech Eng, , 2003. (6) (125): p. 893-901

3. Abreu, E., Leigh, D., Derwin, K A.,, Effect of altered mechanical load conditions on the structure and function of cultured tendon fascicles. Journal of Orthopaedic Research, 2008. 26(3): p. $364-373$.

4. Lin, T.W., Cardenas, L., Soslowsky, L J.,, Biomechanics of tendon injury and repair. Journal of Biomechanics 2004. 37(6): p. 865-877.

5. Aspenberg, P., Stimulation of tendon repair: mechanical loading, GDFs and platelets. A mini-review. International Orthopaedics, 2007. 31(6): p. 783-789. 
6. Rees, J., Lichtwark, GA., Wolman, RL., and Wilson, AM., , The mechanism for efficacy of eccentric loading in Achilles tendon injury; an in vivo study in humans. .

Rheumatology 2008. 47(10): p. 1493-1497.

7. Alfredson, H., Pietila, T., Jonsson, P., Lorentzon, R.,, Heavy-load eccentric calf muscle training for the treatment of chronic Achilles tendinosis. . Am J Sports Med, 1998. 26(3): p. 360-6.

8. Takai, S., Woo, S. L., Horibe, S., Tung, D. K., Gelberman, R. H., , The effects of frequency and duration of controlled passive mobilization on tendon healing. J Orthop Res,, 1991. 9(5): p. 705-13.

9. Screen, H., Shelton, JR., Bader, DL., D., Lee, DA., , Cyclic tensile strain upregulates collagen synthesis in isolated tendon fascicles. Biochemical and Biophysical Research Communications, 2005. 336(2): p. 424-429.

10. Arnoczky, S., Tian, T., Lavagnino, M., Gardner, K.,, Ex vivo static tensile loading inhibits MMP-1 expression in rat tall tendon cells through a cytoskeletally based mechanotransduction mechanism. J. Journal of Orthopaedic Research, 2004. 22(2): p. $328-333$.

11. Lavagnino, M.H., J.A., Arnoczky, SP., Hoonjan, A., Torzilli, PA.,, Effect of amplitude and frequency of cyclic tensile strain on the inhibition of MMP-1 $m R N A$ expression in tendon cells: An in vitro study. . Connective Tissue Research, 2003. 44(3-4): p. 181-187.

12. Chen, Y., Wang, CJ., Yang, KD., Kuo, YR., Huang, HC., Huang, YT., Sun, YC., Wang, FS.,, Extracorporeal shock waves promote healing of collagenase-induced Achilles tendinitis and increase TGF-betal and IGF-I expression. . J Orthop Res, 2004. 22(4): p. 854-61. 
13. Asundi, K., Kursa, K., Lotz, J., Rempel, DM.,, In vitro system for applying cyclic loads to connective tissues under displacement or force control. Ann Biomed Eng,, 2007. 35(7): p. $1188-95$.

14. Kondratko-Mittnacht, J., Lakes, R., Vanderby Jr, R.,, Shear loads induce cellular damage in tendon fascicles. J Biomech., 2015. 48(12): p. 3299-3305.

15. McCarthy, D., Tramaglini, DM., Chan, SS., Schmidt, CC., Sotereanos, DG., Herndon, JH.,, Effect of partial laceration on the structural properties of the canine FDP tendon: an in vitro study. . J Hand Surg Am, 1995. 20(5): p. 795-800.

16. Gelberman, R., Manske, PR., Vande Berg, JS., Lesker, PA., Akeson, WH., 1984. 2(1): p., Flexor tendon repair in vitro: a comparative histologic study of the rabbit, chicken, dog, and monkey. J Orthop Res, 1984. 2(1): p. 39-48.

17. Tanaka, H., Manske, PR., Pruitt, DL., Larson, BJ., , Effect of cyclic tension on lacerated flexor tendons in vitro. J Hand Surg Am, 1995. 20(3): p. 467-73.

18. Grigg, N., Wearing, SC., O'Toole, JM., Smeathers, JE.,, Achilles tendinopathy modulates force frequency characteristics of eccentric exercise. Med Sci Sports Exerc., 2013. 45(3): p. $520-6$.

19. Legerlotz, K., Jones, GC., Screen, HR., Riley, GP., , Cyclic loading of tendon fascicles using a novel fatigue loading system increases interleukin-6 expression by tenocytes. Scand J Med Sci Sports,, 2013. 23(1): p. 31-7.

20. Mass, D., Tuel, RJ., Labarbera, M., Greenwald, DP., , Effects of constant mechanical tension on the healing of rabbit flexor tendons. . Clin Orthop Relat Res, , 1993. 296: p. $301-6$. 
21. Rieder, F., Wiesinger, HP., Kösters, A., Müller, E., Seynnes, OR.,, Whole-body vibration training induces hypertrophy of the human patellar tendon Scand J Med Sci Sports,, 2015: p. doi: 10.1111/sms.12522.

22. Sandhu, E., Miles, JD, Dahners, LE, Keller, BV, Weinhold, PS.,, Whole body vibration increases area and stiffness of the flexor carpi ulnaris tendon in the rat. . J Biomech 2011. 44(6): p. 1189-1191.

23. Keller, B., Davis, ML., Thompson, WR., Dahners, LE., Weinhold, PS.,, Varying whole body vibration amplitude differentially affects tendon and ligament structural and material properties J Biomech, 2013. 46(9): p. 1496-500.

24. Adekanmbi, I., Baboldashti, NZ., Yapp, C., Franklin, S., Thompson, MS., , A novel in vitro loading system for high frequency loading of cultured tendon fascicles. Med Eng Phys, 2013. 35(2): p. 205-10.

25. Fung, D., Wang, VM., Andarawis-Puri, N., Basta-Pljakic, J., Li, Y., and D. Laudier, Sun, HB., Jepsen, KJ., Schaffler, MB., Flatow, EL.,, Early response to tendon fatigue damage accumulation in a novel in vivo model. Journal of Biomechanics 2010. 43(ww): p. 274 279.

26. Grant, T., Yapp, C., Chen, Q., Czernuszka, JT., and Thompson, MS.,, The Mechanical, Structural, and Compositional Changes of Tendon Exposed to Elastase. Annals of Biomedical Engineering, , 2015. 43(10): p. 2477-2486.

27. McCarthy, D., Tramaglini, DM., Chan, SS., Schmidt, CC., Sotereanos, DG., Herndon, JH.,, Effect of partial laceration on the structural properties of the canine FDP tendon: an in vitro study. J Hand Surg Am, 1995. 20(5): p. 795-800. 
28. Asundi, K., Kursa, K., Lotz, J., Rempel, DM., In vitro system for applying cyclic loads to connective tissues under displacement or force control. Ann Biomed Eng, 2007. 35(7): p. $1188-95$.

29. Gelberman, R., Manske, PR., Vande Berg, JS., Lesker, PA., Akeson, WH.,, Flexor tendon repair in vitro: a comparative histologic study of the rabbit, chicken, dog, and monkey. J Orthop Res, 1984. 2(1): p. 39-48.

30. Takai, S., Woo, S. L., Horibe, S., Tung, D. K., Gelberman, R. H.,, The effects of frequency and duration of controlled passive mobilization on tendon healing. J Orthop Res, 1991. 9(5): p. 705-13.

31. Andersson, T., Eliasson P., Aspenberg P.,, Tissue memory in Healing Tendons: Short loading episodes stimulates Healing. J. Appl Physiol, 2009. 107(2): p. 417-421.

32. Carpenter, J., Hankenson, KD.,, Animal models of tendon and ligament injuries for tissue engineering applications. Biomaterials, 2004. 25(9): p. 1715-22.

33. Carpenter, J., Thomopoulos, S., and L.J. Soslowsky, Animal models of tendon and ligament injuries for tissue engineering applications. Clin Orthop Relat Res, 1999(367 Suppl): p. S296-311. 


\section{Figure list}

Figure 1 Illustration of apparatus and set-up used for application of scratch damage in RTTF Pipette and DMEM

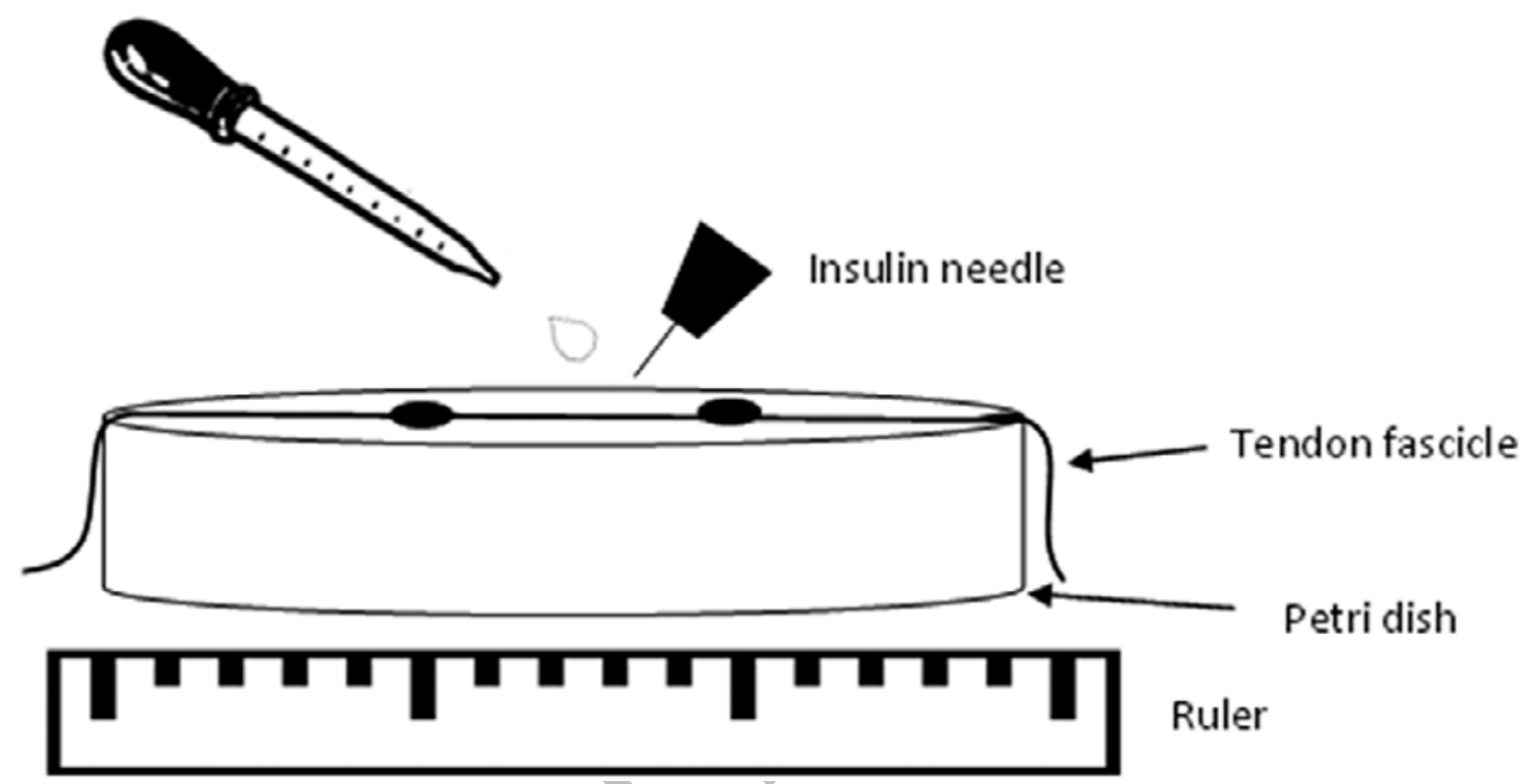


Figure 2 Load-time charts for individual RTTFs at applied loading frequencies of a.) $1 \mathrm{~Hz}$ and b.) $20 \mathrm{~Hz}$. In the charts, sinusoidal and straight profiles represent the cyclic and static load respectively.
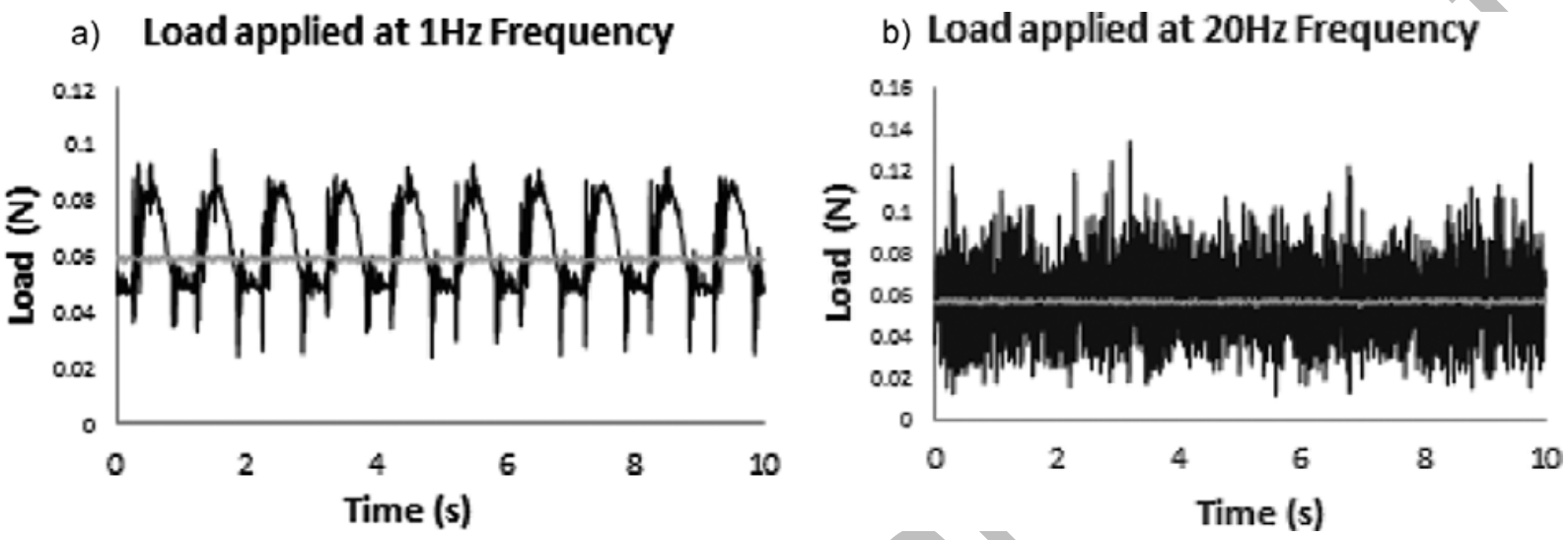
Figure 3: Quantification of live and dead cells in fresh and 7 day cultured tendon groups.

Stained samples were used to perform cell counts in a centre region (area: $1.7 \mathrm{~mm} \times 1.3 \mathrm{~mm}$ ) of each tendon fascicle image. The quantity of live and dead cells is expressed as a percentage of the total number of cells in each tendon group $(n=2)$. Un-intact, Fresh-D, Un-D, Static-D, $1 \mathrm{~Hz}-$ D, 20Hz-D, denote Unloaded intact, Fresh damaged, unloaded damaged, Static loaded damaged, Low frequency cyclic loaded damaged, and HFLM cyclic loaded damaged tendon groups respectively

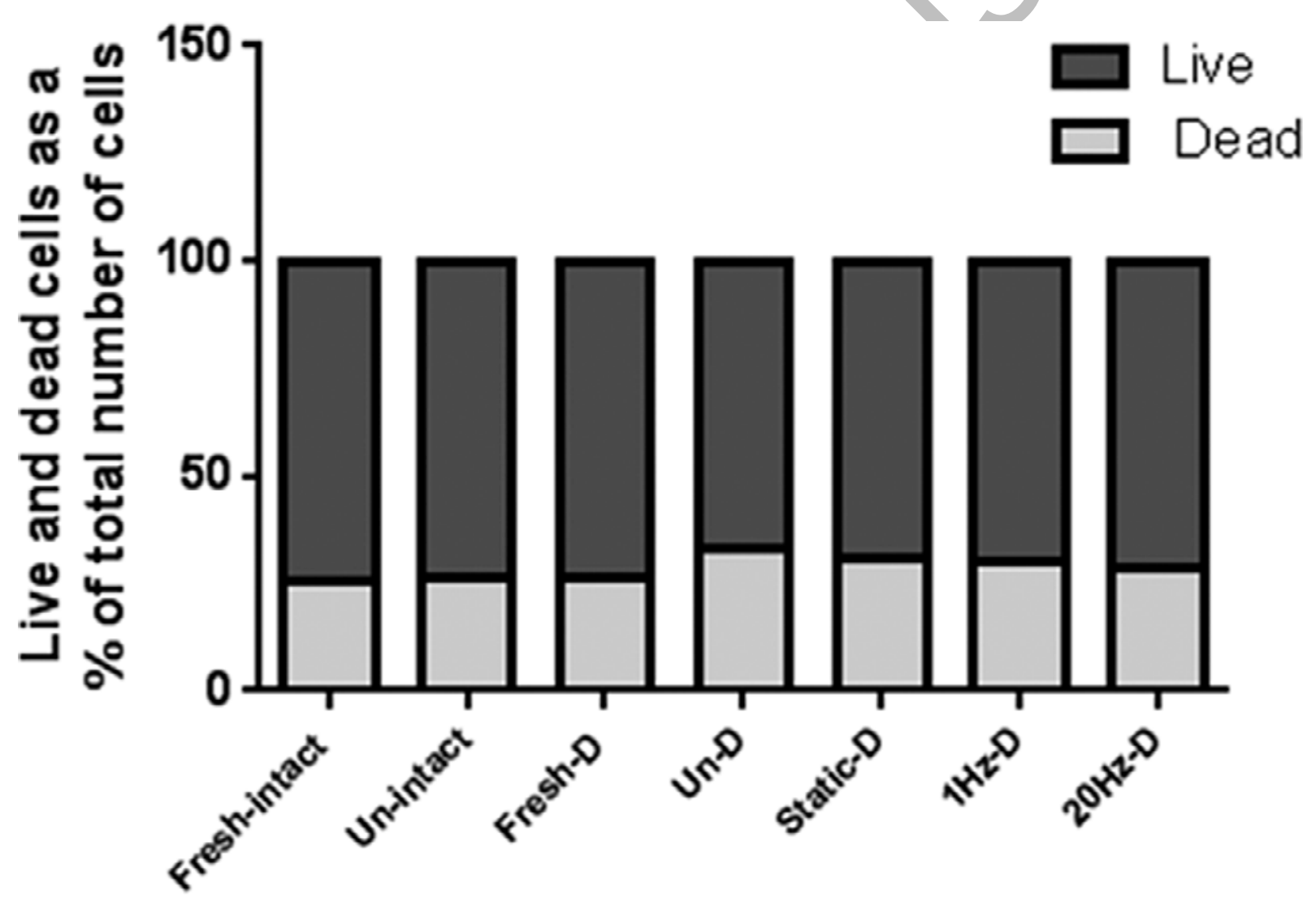

Tendons Groups 
Figure 4: Second Harmonic Generation (SHG) images of Un-D tendon fascicle showing the damage severity after application of a longitudinal scratch. The damaged region is highlighted by the area between the dashed lines.

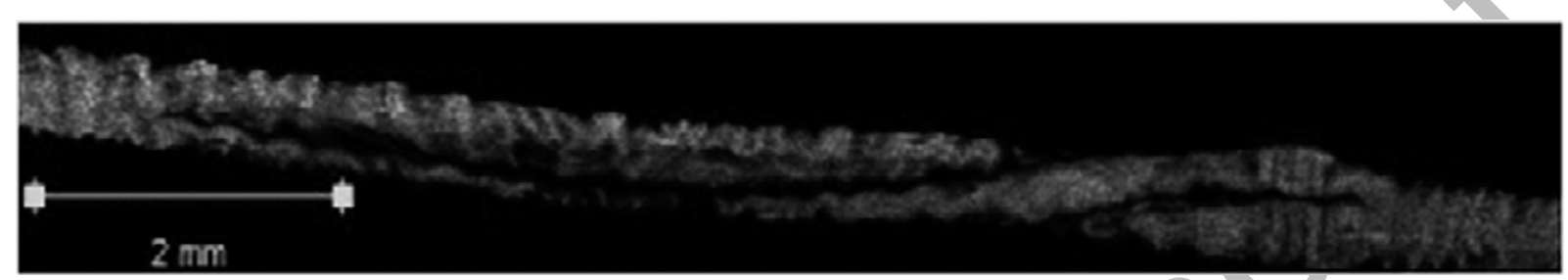


Figure 5: Mechanical validation of injury model showing tangent modulus in unloaded damaged and unloaded intact tendons A.) Immediately (day 0) and B.) 7 days after culture under conditions of no load. Four RTTFS were assessed in each tendon group. Values shown are the mean and the standard deviation, expressed a fraction of the intact unloaded tendon. $*$ denotes a significant difference $(\mathrm{p}<0.05)$ with intact tendons (Student's t-test).
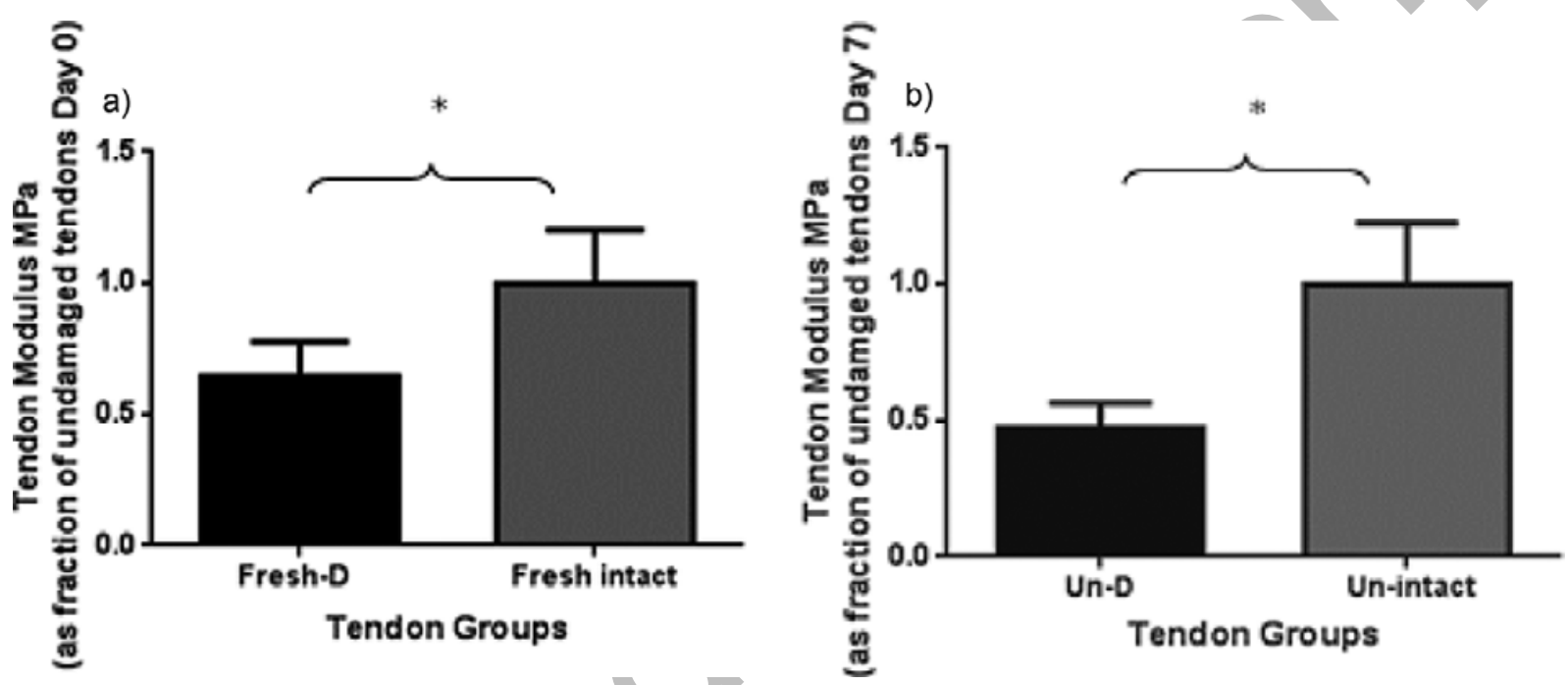
Figure 6: Biological validation of injury model showing metabolic activity in unloaded damaged and unloaded intact tendons A.) Immediately (day 0) and B.) 7 days after culture under conditions of no load. Four RTTFs were used for assessment at each time point and mean relative fluorescence units (RFUs) for each group are expressed as fractions of intact tendon groups at each time point. * denotes a significant difference $(\mathrm{p}<0.05)$ with intact tendons (Student's t-test).
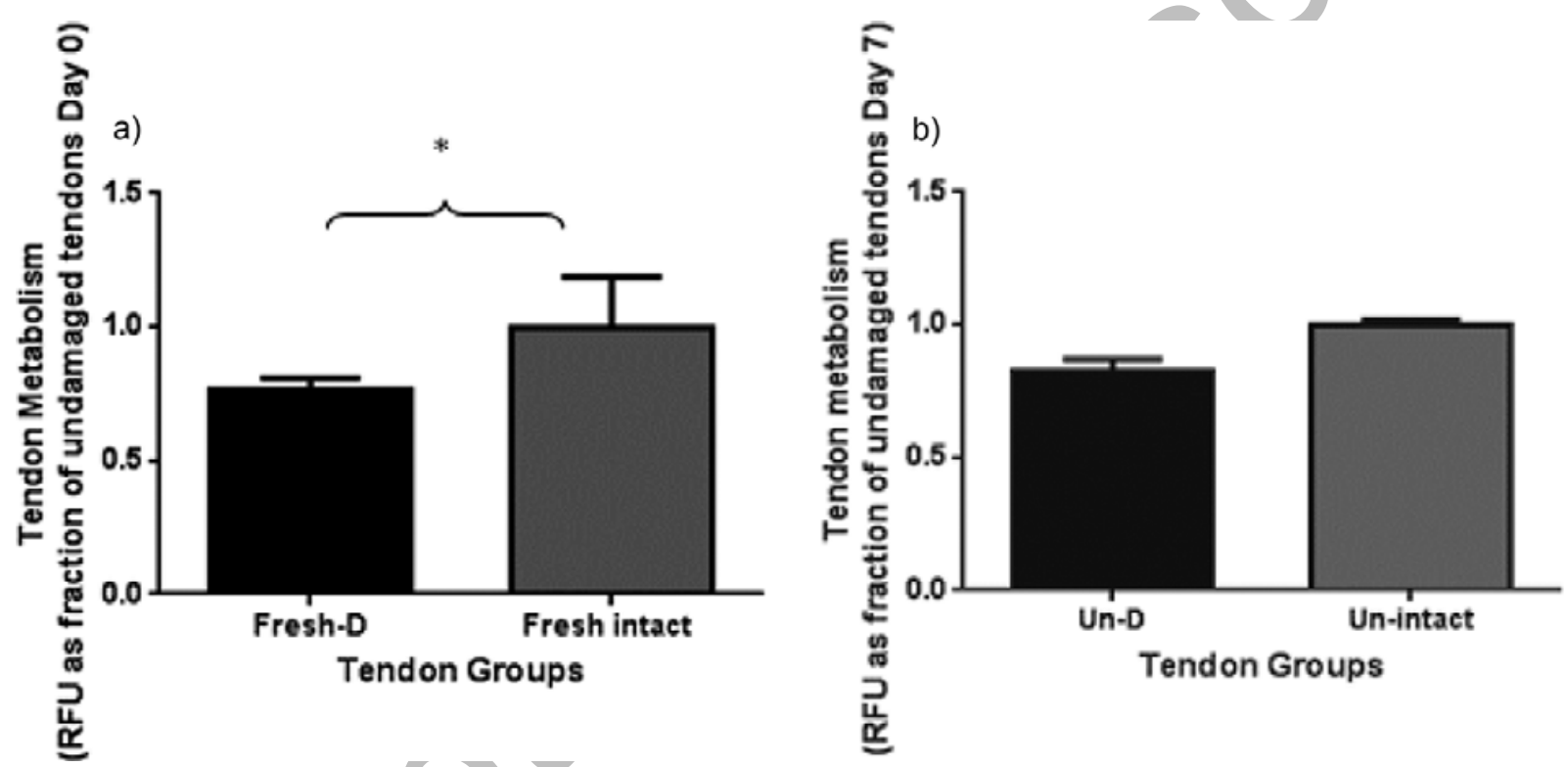
Figure 7: Mean tangent modulus in fresh intact, unloaded and damaged tendon groups under various mechanical loading conditions. $*(\mathrm{p}<0.05)$ and $* * *(\mathrm{p}<0.0001)$ denote a significant difference between unloaded damaged tendon groups. HFLM-D tendon showed significantly higher modulus than Static-D tendons $* *(\mathrm{p}<0.0005)$. The bars shown represent the standard deviation.

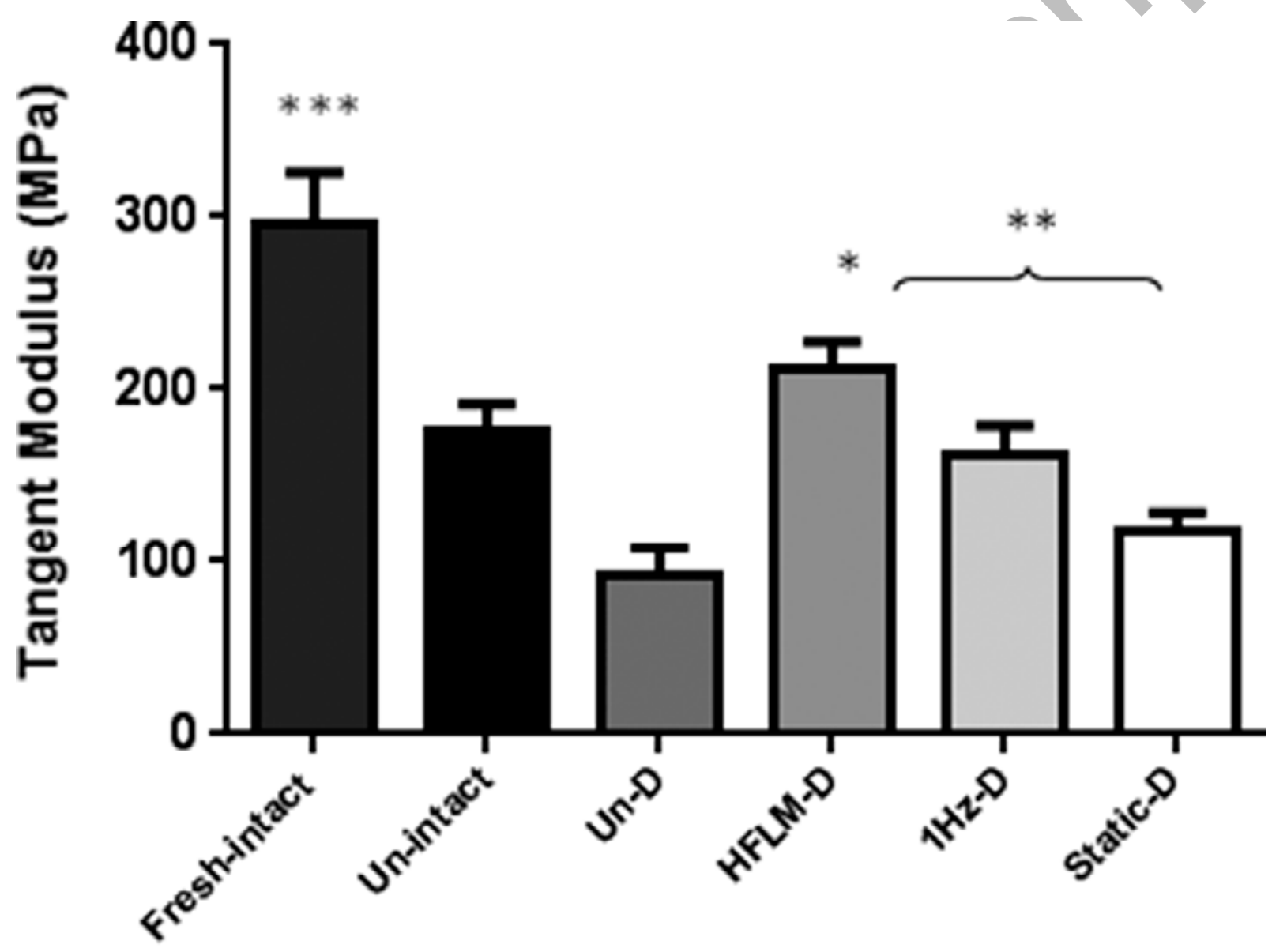

Tendon Groups 
Figure 8: Metabolism of damaged RTTF groups under static, low frequency cyclic, and HFLM mechanical loading conditions; with fresh damaged, fresh intact and unloaded intact RTTFs serving as complimentary controls. *Denotes a significant difference $(p<0.05)$ with unloaded damaged (Un-D) tendons.

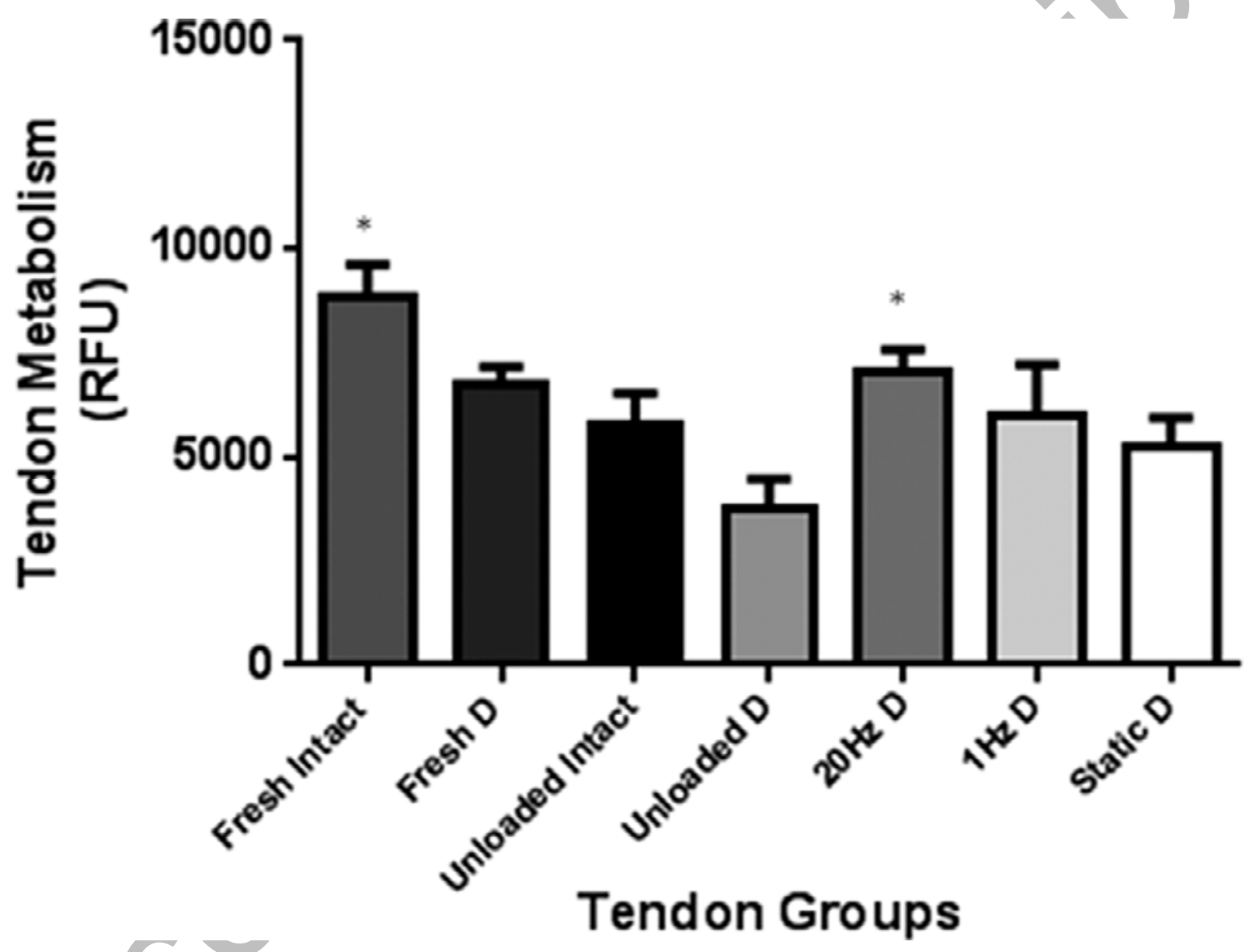


Table 1 Tendon groups and the number of tendon fascicles assessed

\begin{tabular}{|c|c|c|c|c|c|c|c|}
\hline \multirow{2}{*}{$\begin{array}{c}\text { Experiment } \\
\text { (culture time) }\end{array}$} & \multicolumn{6}{|c|}{ Number of RTTFs in tendon experiments } \\
\cline { 2 - 8 } & Fresh-D & Fresh intact & Un-D & Un-intact & Static-D & 1Hz-D & 20Hz-D \\
\hline Tendon viability (7 day) & 2 & 2 & 2 & 2 & 2 & 2 & 2 \\
\hline Damage length (3 day) & - & - & 3 & - & - & - & - \\
\hline $\begin{array}{c}\text { Mechanical damage severity } \\
\#(0,7 \text { day) }\end{array}$ & $4 \#$ & $4 \#$ & $4 \#$ & $4 \#$ & - & - & - \\
\hline $\begin{array}{c}\text { Biological damage severity } \\
\#(0,7 \text { day) }\end{array}$ & $4 \#$ & $4 \#$ & $4 \#$ & $4 \#$ & - & - & - \\
\hline $\begin{array}{c}\text { HFLM effect on modulus } \\
\text { (7 day) }\end{array}$ & - & 8 & 8 & 8 & 8 & 7 & 9 \\
\hline $\begin{array}{c}\text { HFLM effect on metabolism } \\
\text { (7 day) }\end{array}$ & 5 & 5 & 9 & 8 & 8 & 7 & 7 \\
\hline
\end{tabular}

'Un' denotes unloaded tendon groups and ' $\mathrm{D}$ ' denotes damaged tendon. \# indicates tendon fascicles used for validation experiments where damaged and undamaged controls were taken from the same fascicle. 